\title{
Relationship among Preoperative, Pre and Postinduction Blood Pressure in Normotensive and Hypertensive Patients
}

Ricardo Caio Gracco De Bernardis ${ }^{1,2}$ (D), Helena Knebel Vieira De Souza ${ }^{1}$ iD, Jesse Abimael Infante Izquierdo $^{1}$ (D), Mônica Maria Siaulys ${ }^{1,3,4}$ (D), Mauro Prado Da Silva ${ }^{1,3,4}$ (D), Marcel Veronez Vitoreli ${ }^{1}$ (D), Pedro Ferretti Pinheiro ${ }^{1,4}$ (D), Silvio Julio Bittencourt Yamamura ${ }^{1,4}$ (D) and Ligia Andrade Da Silva Telles Mathias $^{1,3,5^{*}}$ (D)

${ }^{1}$ Anesthesia Department, Itapecerica of Serra General Hospital, Itapecerica of Serra, Brazil

${ }^{2}$ Anesthesia Department, Maria Zelia Hospital, São Paulo, Brazil

${ }^{3}$ Anesthesia Department, Santa Joana Corporation, São Paulo, Brazil

${ }^{4}$ Anesthesia Department, Santa Casa of São Paulo, São Paulo, Brazil

${ }^{5}$ Anesthesia Department, College of Medical Sciences of Santa Casa of São Paulo, São Paulo, Brazil

\begin{abstract}
Objectives: This study aimed to verify the relationship between blood pressure (BP) obtained during preoperative evaluation at preinduction and compared to BP at $20 \mathrm{~min}$ after induction for normotensive and hypertensive patients.

Methods: Data from patients who underwent elective surgical procedures from July 2018 to September 2019 were retrospectively extracted and analyzed. The data included patient characteristics (age, sex, weight, height, body mass index (BMI)), physical status (ASA classification), comorbidities, medications in use, and mean arterial BP (MAP) recorded preoperatively (MAPpre-op), before induction of anesthesia (MAPpre-ind), and $20 \mathrm{~min}$ after induction (MAPpost-ind).

Results: A total of 1026 patients were selected, 341 were included in the final analysis (normotensive patients (GNT $n=$ 203), treated hypertensive patients (GTREAT $n=98$ ), untreated hypertensive patients (GNO TREAT $n=40$ ). There was an increase in the median MAP from preoperation to preinduction in the total sample and the three groups (GNT, GTREAT, and GNO TREAT). There was a statistically significant reduction in MAPpost-ind concerning MAPpre-ind and MAPpre-op in the three groups. The three groups' comparison showed a statistically significant difference between MAPpost-ind and MAPpre-ind and between MAPpost-ind and MAPpre-op.

Conclusions: Our study showed that normotensive and hypertensive patients showed a significant BP increase at the preinduction time point. Treated and untreated hypertensive patients had a more considerable increase in preinduction $\mathrm{BP}$ and BP reduction at $20 \mathrm{~min}$ after anesthesia induction than normotensive patients. These changes were more significant in hypertensive patients without treatment.
\end{abstract}

\section{Keywords}

Preoperative, Postinduction, Blood pressure, Hypertension, Anesthesia

\section{Introduction}

Changes in intraoperative blood pressure (BP) are frequent, especially hypotension, during noncardiac and cardiac surgery with general anesthesia. Adverse intra and postoperative outcomes involving postoperative myocardial ischemia and myocardial infarction, ischemic renal injury, stroke, postoperative cognitive decline, and death are related to intraoperative hypotension [1-9]. Although hypertension alone is not regularly correlated with perioperative cardiovascular complications, it may induce intra-operative hypotension and tachycardia, which may be associated with perioperative morbimortality $[10,11]$.

However, there is no consensus regarding whether intraoperative hypotension $(\mathrm{IOH})$ should be considered when the patient has a BP below a threshold value or presents a reduction in baseline BP values, neither there is a consensus been reached regarding the specific importance of postinduction hypotension, which is estimated to occur at up to $20 \mathrm{~min}$ after induction (BPpost-ind) [5,6,12-21]. 
Many studies about $\mathrm{IOH}$ evaluate the decrease in baseline BP compared to preinduction BP. However, others define baseline BP as the BP measured preoperatively (BPpre-op) $[7,8,12,14-18]$. The performance of hypertensive patients regarding $\mathrm{IOH}$, BPpre-op, and BPpre-ind, and, therefore, the definition of baseline BP has been minimally studied $[7,8,12,14-18]$.

The clinical relevance of defining the appropriate reference time point for baseline BP lies in the importance of diagnosing intraoperative hypertension or hypotension during the anesthetic-surgical procedure and taking the proper procedures according to each of these situations, which is especially crucial for hypertensive patients.

The present study's objective was to verify the relationship between preoperative and preinduction BP and compare to $\mathrm{BP}$ at 20 min after induction for normotensive and hypertensive patients.

\section{Materials and Methods}

The Institutional Ethics Committee approved the protocol of this retrospective observational study. Data from patients who underwent elective surgical procedures from July 2018 to September 2019 were retrospectively extracted and analyzed.

\section{Inclusion and exclusion criteria}

Patients were eligible for 18 years of age or older; were American Society of Anesthesiologists (ASA) class I, II or III; had preinduction and intraoperative BPs measured in the supine position and had undergone elective surgery under general anesthesia. The following patients were excluded: Those who were pregnant, had implantable devices, had received preanesthetic medication, or had undergone neurosurgery and cardiac surgery.

\section{Data collection}

The data included patient characteristics (age, sex, weight, height, body mass index (BMI)), physical status (ASA classification), comorbidities, medications in use, and BPs recorded preoperatively, before induction of anesthesia, and $20 \mathrm{~min}$ after induction (MAPpost-ind).

The BPpre-op was obtained in the preanesthetic evaluation clinic (at a median of 2 weeks before surgery) after the patient had been sitting for at least five min [4].

Preoperative BP measurements were performed with the patients in a seated position using an automated noninvasive BP device (oscillometric measurement) with the appropriate cuff for the patient's arm width. The systolic BP (SBP) and diastolic $B P(D B P)$ values were measured, and the mean arterial $\mathrm{BP}$ (MAP) value was calculated using the formula (SBP + 2DBP/3).

$\mathrm{BP}$ was measured with the patient in the supine position in the operating room, and the noninvasive BP module (NIBP) of the Dräger multiparameter monitor was used. The BP values were automatically stored in the anesthesia information management system, and the measured MAP was included; measurements were usually taken every three to five min.
The BPpre-ind was the BP at the time of the patient's arrival in the operating room before anesthesia induction [14].

All negative BP values, DBP values greater than the corresponding MAP values and MAP values greater than the corresponding SBP values were considered artifacts and were analyzed as missing data. As the study included only patients undergoing elective surgery, DBP values less than 40 or greater than $100 \mathrm{mmHg}$, MAP values less than 50 or greater than $130 \mathrm{mmHg}$, and SBP values less than 80 or greater than 200 $\mathrm{mmHg}$ were considered outside the normal physiological range and were analyzed as missing data.

\section{Definition of hypertension and hypotension}

Patients who reported being hypertensive, regardless of whether they were taking antihypertensive medication, were considered hypertensive, even when their BP was within normal limits.

As there is no single threshold proposed in the literature for defining hypertension and hypotension, thresholds of SBP $\geq 140 \mathrm{mmHg}, \mathrm{DBP} \geq 90 \mathrm{mmHg}$ and MAP $\geq 100 \mathrm{mmHg}$ were chosen to indicate hypertension; a greater than $30 \%$ reduction of MAPpre-ind or absolute MAP $<65 \mathrm{mmHg}$ were chosen to indicate intraoperative hypotension $(\mathrm{IOH}) 20 \mathrm{~min}$ after induction (IOHpost-ind) [4,13,18-20].

\section{Statistical Analysis}

The descriptive results are presented as medians and $25^{\text {th }}$ and $75^{\text {th }}$ percentiles for continuous data and as frequencies and absolute percentages for categorical data. A separate analysis of normotensive (GNT) and hypertensive patients was performed by evaluating those under treatment (GTREAT) and those without treatment (GNO TREAT), and the MAP values are presented as box plots.

The Mann-Whitney test or Fisher's test and c2 test were used to compare two groups, and the $\mathrm{c} 2$ test, Friedmann test, or Kruskal-Wallis test were used when more than two groups were evaluated. If a statistically significant difference was found between the groups, the Dunn test was used for the pairwise comparison.

A linear correlation test with Pearson's coefficient was performed for MAPpre-ind vs. MAPpre-op and MAPpost-ind vs. MAPpre-ind.

*Corresponding author: Ligia Andrade da Silva Telles Mathias, Anesthesia Department, Itapecerica of Serra General Hospital, Itapecerica of Serra, Brazil; Anesthesia Department, Santa Joana Corporation, São Paulo, Brazil; Anesthesia Department, College of Medical Sciences of Santa Casa of São Paulo, São Paulo, Brazil

Accepted: December 21, 2020

Published online: December 23, 2020

Citation: Bernardis RCGD, Souza HKVD, Izquierdo JAI, et al. (2020) Relationship among Preoperative, Pre and Postinduction Blood Pressure in Normotensive and Hypertensive Patients. J Clin Anesth Pain Manag 4(2):143-149 
Citation: Bernardis RCGD, Souza HKVD, Izquierdo JAl, et al. (2020) Relationship among Preoperative, Pre and Postinduction Blood Pressure in Normotensive and Hypertensive Patients. J Clin Anesth Pain Manag 4(2):143-149

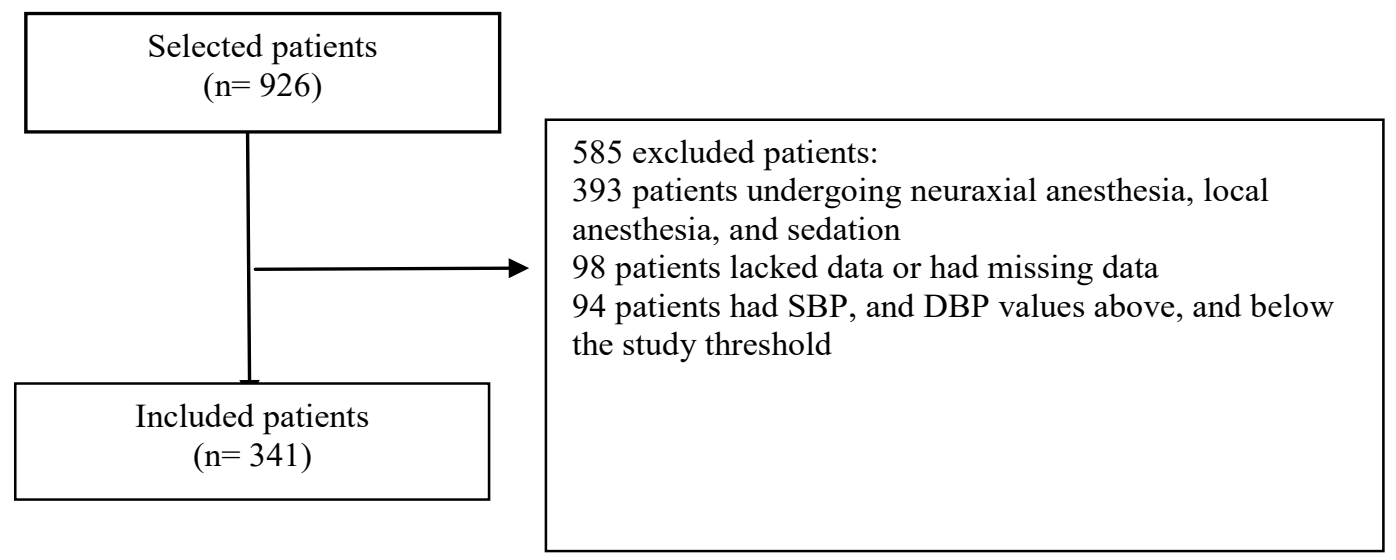

Figure 1: Flow chart illustrating the inclusion of patients and the reasons for exclusion.

Table 1: Patient characteristics.

\begin{tabular}{|c|c|c|c|c|c|}
\hline & Total number of patients & GNT & GTREAT & GNO TREAT & $\mathbf{P}$ \\
\hline $\mathrm{N}$ & 341 & 203 & 98 & 40 & \\
\hline Age (years) & $44(33-58)$ & $36(29-46.5)$ & $55(44-68)$ & $57(46-76)$ & $<0.0001^{*}$ \\
\hline $\operatorname{Sex}(F)$ & $178(52.2)$ & $88(43.4)$ & $65(66.3)$ & $25(62.5)$ & $<0.0001^{* *}$ \\
\hline $\mathrm{BMI}\left(\mathrm{kg} \cdot \mathrm{m}^{-2}\right)$ & $26.8(24.2-29.9)$ & $25.7(23.9-28.4)$ & $27.9(25.6-32.0)$ & $30.5(23.7-34.1)$ & $<0.0001^{*}$ \\
\hline \multicolumn{6}{|c|}{ Physical status } \\
\hline ASA I & 102 (29.9) & $117(57.6)$ & 0 & 0 & $<0.0001^{* *}$ \\
\hline ASA II & $223(65.6)$ & $83(40.9)$ & $87(88.8)$ & $30(75.0)$ & $<0.0001^{* *}$ \\
\hline ASA III & $16(4.5)$ & $3(1.5)$ & $11(11.2)$ & $10(25.0)$ & $<0.0001^{* *}$ \\
\hline
\end{tabular}

Data are presented as the median $\left(25^{\text {th }}\right.$ and $75^{\text {th }}$ percentiles) or number of patients (\%).

GNT: Normotensive patients; GTREAT: Treated hypertensive patients; GNO TREAT: Hypertensive patients without treatment; F: Female; BMI: Body mass index; ASA: Physical status (American Society of Anesthesiologists class); *: Kruskal-Wallis test for GNT, GTREAT and GNO TREAT;

${ }^{* *}$ : Chi-square test for GNT, GTREAT and GNO TREAT.

Table 2: Preinduction, preoperative, 20 min postinduction BP in the three groups (GNT, GTREAT, and GNO TREAT).

\begin{tabular}{|l|l|l|l|l|l|}
\hline & Total number of patients & GNT & GTREAT & GNO TREAT & P \\
\hline N & 341 & 203 & 98 & 40 & \\
\hline MAPpre-op $(\mathrm{mmHg})$ & $93(84-102)$ & $89(82-93)$ & $102(93-108)$ & $107(100-110.5)$ & $<0.0001^{*}$ \\
\hline MAPpre-ind $(\mathrm{mmHg})$ & $100(93-110)$ & $96(89-101)$ & $109(100-117)$ & $117.5(110-121)$ & $<0.0001^{*}$ \\
\hline MAPpost-ind $(\mathrm{mmHg})$ & $86(73.5-88)$ & $81(76-88)$ & $87(79-96)$ & $74.5(64-90)$ & $<0.0001^{*}$ \\
\hline $\mathrm{P}$ & $<0.0001^{* *}$ & $<0.0001^{* *}$ & $<0.0001^{* *}$ & $<0.0001^{* *}$ \\
\hline
\end{tabular}

Data are presented as the median $\left(25^{\text {th }}\right.$ and $75^{\text {th }}$ percentiles) and the respective $p$ values of the Kruskal-Wallis ${ }^{*}$ and Friedman's tests ${ }^{* *}$.

GNT: Normotensive patients; GTREAT: Treated hypertensive patients; GNO TREAT: hypertensive patients without treatment; MAPpre-ind: MAP preinduction; MAPpre-op: Preoperative MAP; MAPpost-ind: MAP $20 \mathrm{~min}$ after anesthetic induction.

Microsoft Excel 365 (Microsoft Corp., USA) was used for data management, and GraphPad Prism 8.4.3 (GraphPad, USA) was used for statistical analysis. All statistical tests were bilateral, and $\mathrm{P}$ values less than 0.05 were considered statistically significant.

\section{Results}

A total of 1026 patients were selected during the study period (Figure 1); after their medical records were analyzed, 341 were included in the final analysis. The final sample (341 patients) as a whole was analyzed, and separate analyses were performed for normotensive patients (GNT $n=203$ ), treated hypertensive patients (GTREAT $n=98$ ), and untreated hypertensive patients (GNO TREAT $n=40$ ).

The characteristics of the patients are shown in Table 1. Among the comorbidities, the most frequent were hypertension, 138 (40.5\%), followed by diabetes mellitus, 61 (17.9\%). Of the hypertensive patients, 98 (59.2\%) were treated with different antihypertensive medications (often combinations of angiotensin-converting enzyme inhibitors (26), calcium 
channel blockers (19), angiotensin II receptor antagonists (35), beta-blockers (13), and diuretics (23)), and $40(27.4 \%)$ were unaware that they were hypertensive and/or did not take any antihypertensive medication. The surgeries comprised 201 (59\%) digestive tract surgeries, 79 (23\%) orthopedic surgeries, $46(13.5 \%)$ gynecological surgeries, and 15 (4.5\%) vascular surgeries.

The preinduction $\mathrm{BP}$, preoperative $\mathrm{BP}$, and $\mathrm{BP}$ at $20 \mathrm{~min}$ postinduction are shown in Table 2.

\section{Comparison of preoperative and preinduction BP}

There was an increase in the median MAP from preoperation to preinduction in the total sample and each of the three groups (GNT, GTREAT, and GNO TREAT).

There was a weak statistically significant correlation between the MAPpre-op and MAPpre-ind ( $p<0.0001$ ), with Pearson correlation coefficients of $0.5569(95 \% \mathrm{Cl}, 0.7069$ $0.7810), 0.3285$ (95\% Cl 33.04-48.28), 0.5049 (95\% Cl 9.39831.72 ) and 0.012 (95\% Cl 55.67-126.9) for the total sample and GNT, GTREAT, and GNO TREAT, respectively.

In the total sample, there was variation between the median differences in MAPpre-op and MAPpre-ind $(8 \mathrm{mmHg})$. A total of 294 patients (86.2\%) had a MAPpre-ind that was higher than their MAPpre-op; of these, 62 (21.1\%) had a difference (pre-ind - pre-op) greater than $10 \mathrm{mmHg}$. Only $20 \mathrm{pa}-$ tients (5.9\%) had a negative difference in MAP (pre-ind-preop), i.e., a MAPpre-ind that was less than their MAPpre-op.
In the subgroup analysis, the difference between MAPpre-ind and MAPpre-op was greater than or equal to zero (positive) in $84.7 \%, 86.7 \%$, and $92.5 \%$ of the patients in GNT, GTREAT, and GNO TREAT, respectively, with a difference of 11 $\mathrm{mmHg}(95 \% \mathrm{Cl} 8$ to 15$)$ in GNO TREAT, $6 \mathrm{mmHg}(95 \% \mathrm{Cl} 5$ to 7) GNT, and $7 \mathrm{mmHg}(95 \% \mathrm{Cl} 5$ to 8 ) GTREAT (Kruskal-Wallis test, $\mathrm{p}<0.0001)$.

Comparing the three groups of patients showed a statistically significant difference $(p<0.0001)$ in MAPpre-op and MAPpre-ind, with GTREAT presenting a greater increase in MAP compared to GNT and GNO TREAT showing a greater increase in MAP than GTREAT.

In the preoperative period, $58.1 \%$ of the patients in GTREAT and $100 \%$ of the patients in GNO TREAT presented $M A P \geq 100 \mathrm{mmHg}$. In the preinduction period, MAP was $\geq 100$ $\mathrm{mmHg}$ in $27.1 \%$ of the GNT patients, $76.5 \%$ of the GTREAT patients, and $100 \%$ of the GNO TREAT patients.

\section{Comparison of preoperative $\mathrm{BP}$, preinduction $B P$, and BP 20 min after induction}

There was a statistically significant reduction in MAPpost-ind in relation to MAPpre-ind and MAPpre-op ( $p<$ 0.0001 ) in the three groups (Friedman and Dunn tests) (Figure 2).

The comparison of the three groups showed a statistically significant difference between MAPpost-ind and MAPpre-ind and between MAPpost-ind and MAPpre-op ( $p<0.0001$, Kruskal-Wallis and Dunn tests), with GNO TREAT > GTREAT > GNT.

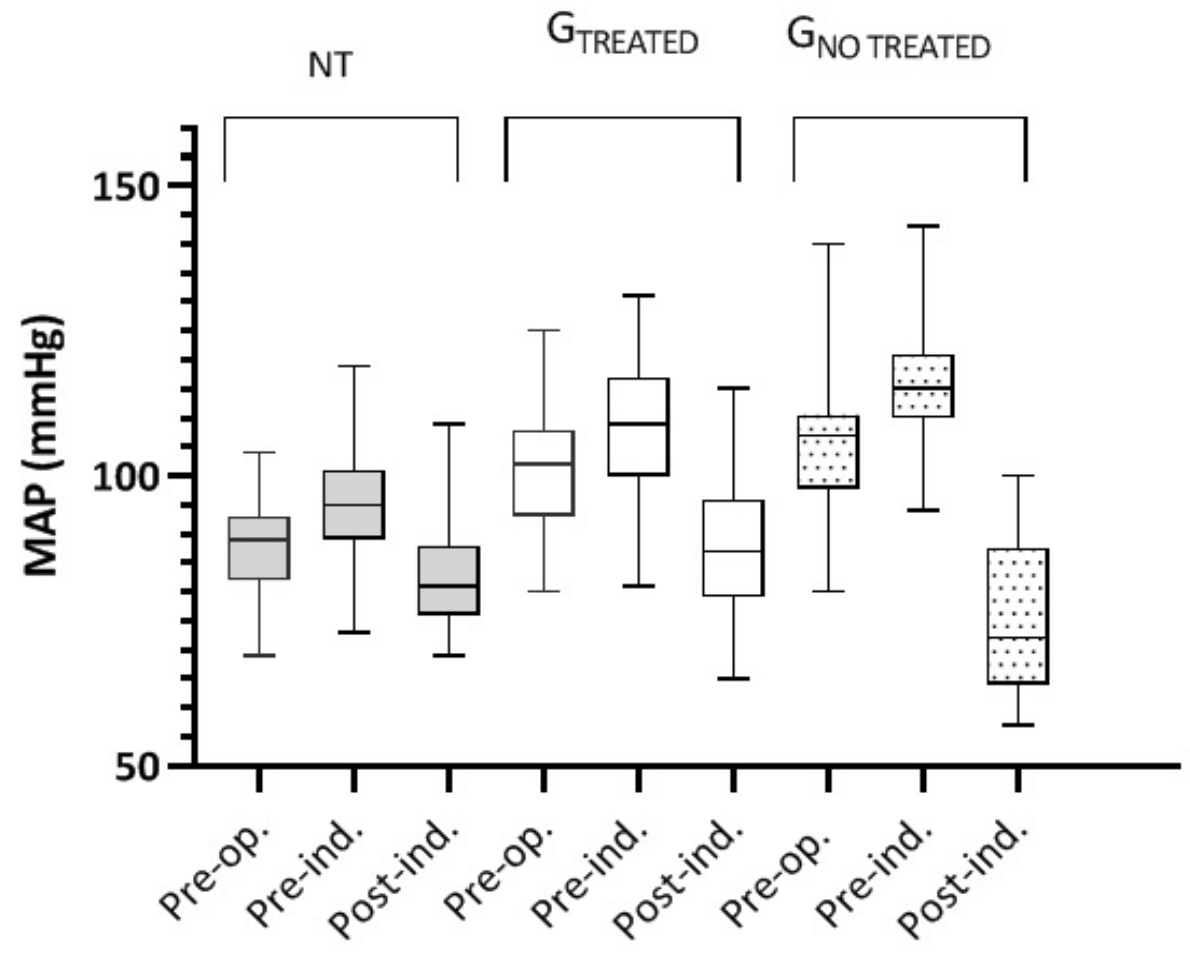

Figure 2: MAPpre-op, MAPpre-ind and MAPpost-ind of normotensive (NT), treated hypertensive (TREATED) and untreated hypertensive (UNTREATED) patients.

Box plots show the median MAP data. The lower and upper edges of the boxes represent the $25^{\text {th }}$ and $75^{\text {th }}$ percentiles. The internal horizontal lines of the boxes represent the medians. 
Citation: Bernardis RCGD, Souza HKVD, Izquierdo JAI, et al. (2020) Relationship among Preoperative, Pre and Postinduction Blood Pressure in Normotensive and Hypertensive Patients. J Clin Anesth Pain Manag 4(2):143-149

The difference between MAPpost-ind and MAPpre-ind was more significant than the difference between MAPpost-ind and MAPpre-op, as predicted (MAPpost-ind - MAPpre-ind: 15, 17, and $23 \mathrm{mmHg}$ for GNT, GTREAT and GNO TREAT; MAPpost-ind - MAPpre-op: 8, 10, and $15 \mathrm{mmHg}$ for GNT, GTREAT, and GNO TREAT, respectively) ( $p<0.0001$ for the three groups, Kruskal-Wallis test, and Dunn test).

There was a greater than $30 \%$ reduction in MAPpost-ind compared to MAPpre-ind in $3.4 \%, 12.2 \%$, and $52.5 \%$ of patients in GNT, GTREAT, and GNO TREAT, respectively.

\section{Discussion}

In this retrospective study, one aim was to evaluate the relationship between preoperative and preinduction MAP in patients who had elective noncardiac surgery with general anesthesia. The difference in MAP in the preoperative period was observed between the three groups. At this time point, $58.2 \%$ of patients in GTREAT were hypertensive, which suggests that they were receiving inadequate treatment, used antihypertensive medication irregularly, or had not taken their medication that day because of the medical consultation [10].

In the preinduction period, all groups had hypertensive patients, including the group of normotensive patients; additionally, there was a significant increase in the number of hypertensive patients in GTREAT from $58.2 \%$ to $76.5 \%$. MAP was higher in GTREAT than in GNT and was even higher in GNO TREAT than in GTREAT. A weak correlation was observed between MAPpre-op and the MAPpre-ind in all groups studied. However, the difference (MAPpre-ind - MAPpre-op) in GNO TREAT was more significant than that of the other two groups $(p<0.0001)$. It is debatable whether this difference is clinically meaningful (11 $\mathrm{mmHg}$ ).

These results suggest that preinduction BP does not reflect baseline BP for either normotensive or hypertensive patients, regardless of treatment, which corroborates other studies $[16,21]$.

A cohort study of 3660 normotensive and hypertensive patients aged over 60 years evaluated the correlation between MAPpre-ind and MAPpre-op and found a mean difference of $11 \mathrm{mmHg}$, which varied from greater than $+10 \mathrm{mmHg}$ to less than $-10 \mathrm{mmHg}$ [15], and a prospective study with 370 normotensive patients between 40 and 65 years of age also found significant variability in the difference between MAPpre-ind and MAPpre-op, with a mean difference of 0 (preoperative BP was calculated as the median of outpatient MAP values during the day and at night) [18].

In the present study, the median difference between MAPpre-ind and MAPpre-op was $8 \mathrm{mmHg}$ for the total sample, $11 \mathrm{mmHg}$ for GNO TREAT, $6 \mathrm{mmHg}$ for GNT, and $7 \mathrm{mmHg}$ for GTREAT. However, there was less variability in the differences than others studies, and only $15.3 \%, 13.3 \%$, and $7.5 \%$ of the patients in GNT, GTREAT, and GNO TREAT presented a negative difference $[15,17]$.

The patients in GTREAT and GNO TREAT were older and more obese and had a higher frequency of ASA II and ASA III physical status (according to the ASA's definition of physical status), which is consistent with the profile of hypertensive patients provided by different authors $[4,14,17]$. However, in the present case series, the patients in GTREAT and GNO TREAT were predominantly female, unlike in other studies $[14,16]$.

No statistical analysis was performed regarding the use of antihypertensive drugs because many patients used combinations of more than one drug (59.2\%), which prevented a separate analysis of each drug's role in the changes in BP $[10,17]$.

We also aimed to compare the preoperative and preinduction MAP to MAP at 20 min after induction for normotensive and hypertensive patients.

There was a statistically significant reduction in MAPpost-ind compared to MAPpre-ind and MAPpre-op in all groups studied in the present study. As expected, the difference was more significant between MAPpost-ind and MAPpre-ind than between MAPpost-ind and MAPpre-op since there was an increase in MAP between the preoperative and preinduction periods. In the comparative analysis among the groups, the reduction was greater in GNO TREAT than in the other two groups and was greater in GTREAT than in the GNT group.

A reduction in MAPpost-ind greater than $30 \%$ ( $1 \mathrm{OH}$ postind) was observed in $52.5 \%$ of GNO TREAT patients, compared to only $12.2 \%$ and $3.4 \%$ of patients in GNT and GTREAT, respectively. The difference was statistically and clinically significant, which can be explained by the correlation between the disease's pathophysiology and the nonuse of antihypertensive medications.

This MAPpost-ind reduction in non-treated hypertensive patients also shows the importance of discriminate this group of patients in the preoperative period and manage them pharmacologically appropriately before the surgery to prevent possible intra and postoperative complications, as previously demonstrated by other authors $[1,2,5,6,16,22]$.

This retrospective study has obvious limitations. The first is that $32 \%$ of patients were excluded due to insufficient data or a lack of records regarding preoperative BP and or preinduction $B P$, which denotes failures to submit patient information. Besides, $10.1 \%$ of patients had BP values above or below the threshold values in this study. These data were considered artifacts and were excluded; however, they could have been accurate measurements. Another limitation is related to the actual use of antihypertensive medications on the day of surgery. Because this was a retrospective study, these information sources were restricted to patient information records, and there was no data on instructions regarding the use of medications on the day of surgery. Because it was a retrospective study, a convenience sample was used; the sample may have been small, since the group of hypertensive patients without treatment consisted of 40 patients, compared with 98 and 203 in the other groups. Even with a larger sample, this group will always be smaller than the other groups. Preoperative BP was evaluated during the preanesthetic con- 
Citation: Bernardis RCGD, Souza HKVD, Izquierdo JAl, et al. (2020) Relationship among Preoperative, Pre and Postinduction Blood Pressure in Normotensive and Hypertensive Patients. J Clin Anesth Pain Manag 4(2):143-149

sultation; there was no fixed time interval between this time point and the start of the surgery, and the standard, routine timing of the preanesthetic consultation is 2 to 3 weeks before surgery. Intraoperative BP was measured according to intervals determined by the pressure device (between 3 and $5 \mathrm{~min}$ ), which has a lower accuracy than the continuous measurement of BP (invasive BP) with an arterial catheter.

Additionally, the preinduction BP was defined using a single BP measurement taken at the entrance of the operating room; in contrast, other studies used the mean or median of BP measurements taken from entry into the operating room until the beginning of anesthesia induction. This study did not consider intraoperative factors such as the dose of anesthetics, volume of infused fluids, or blood loss in the analysis of $\mathrm{IOH}$. Also, the duration of $\mathrm{IOH}$ episodes was not evaluated, as other studies showed that increases in the duration of the $\mathrm{IOH}$ event increased perioperative morbidity.

\section{Conclusions}

Our study showed that normotensive and hypertensive patients showed a significant increase in BP at the preinduction timepoint, which suggests that the preoperative BP, preferably taken during an outpatient visit, should be considered the baseline BP or reference BP. This study also showed that treated and untreated hypertensive patients should receive special attention regarding BP lability during anesthesia. They had a higher increase in preinduction BP and a greater BP reduction at 20 min after anesthesia induction than normotensive patients. These changes were more remarkable in hypertensive patients without treatment.

\section{Author Contributions}

All authors were fully involved in the production of this manuscript.

Mathias LAST and Bernardis RCG were the intellectual authors of the study.

Mathias LAST, Bernardis RCG, Siaulys MM, da Silva MP, Vitoreli MV, Yamamura SJB and Pinheiro PF contributed to the study conceptualization, formal analysis, methodology, and project administration.

Souza HKV, Izquierdo JAI, Vitoreli MV, Yamamura SJB and Pinheiro PF, Mathias LAST and Bernardis RCG participated in the acquisition, analysis and interpretation of data.

Souza HKV, Izquierdo JAI, Mathias LAST and Bernardis RCG were responsible for manuscript writing.

Mathias LAST, Bernardis RCG, Siaulys MM, da Silva MP, Vitoreli MV, Yamamura SJB and Pinheiro PF critically reviewed the manuscript.

All authors admitted that they are responsible for ensuring that questions related to any part of the study's integrity are judiciously investigated and resolved. All authors participated enough in THIS Paper to take public responsibility for appropriate portions of the content.

\section{Conflicts of Interest}

The authors declare no conflict of interest.

\section{Funding}

This research received no external funding.

\section{References}

1. Hallqvist L, Granath F, Huldt E, et al. (2018) Intraoperative hypotension is associated with acute kidney injury in noncardiac surgery: An observational study. Eur J Anaesthesiol 35: 273-279.

2. Hallqvist L, Mårtensson J, Granath F, et al. (2016) Intraoperative hypotension is associated with myocardial damage in noncardiac surgery: An observational study. Eur J Anaesthesiol 33: 450-456.

3. Monk TG, Bronsert MR, Henderson WG, et al. (2015) Association between intraoperative hypotension and hypertension and 30day postoperative mortality in noncardiac surgery. Anesthesiology 123: 307-319.

4. Aung K, Htay T (2019) Relationship between outpatient clinic and ambulatory blood pressure measurements and mortality. Curr Cardiol Rep 21: 28-35.

5. Salmasi V, Maheshwari K, Yang D, et al. (2017) Relationship between intraoperative hypotension, defined by either reduction from baseline or absolute thresholds, and acute kidney and myocardial injury after noncardiac surgery: A retrospective cohort analysis. Anesthesiology 126: 47-65.

6. Sun LY, Chung AM, Farkouh ME, et al. (2018) Defining an intraoperative hypotension threshold in association with stroke in cardiac surgery. Anesthesiology 129: 440-447.

7. van Waes JA, van Klei WA, Wijeysundera DN, et al. (2016) Association between intraoperative hypotension and myocardial injury after vascular surgery. Anesthesiology 124: 35-44.

8. Wesselink EM, Kappen TH, van Klei WA, et al. (2015) Intraoperative hypotension and delirium after on-pump cardiac surgery. $\mathrm{Br}$ J Anaesth 115: 427-433.

9. Gregory A, Stapelfeldt WH, Khanna AK, et al. (2020) Intraoperative hypotension is associated with adverse clinical outcomes after noncardiac surgery. Anesth Analg.

10. Pfister CL, Govender S, Dyer RA, et al. (2020) A multicenter, cross-sectional quality improvement project: The perioperative implementation of a hypertension protocol by anesthesiologists. Anesth Analg 131: 1401-1408.

11. Crowther M, van der Spuy K, Roodt F, et al. (2018) The relationship between pre-operative hypertension and intra-operative haemodynamic changes known to be associated with postoperative morbidity. Anaesthesia 73: 812-818.

12. Bijker JB, van Klei WA, Kappen TH, et al. (2007) Incidence of intraoperative hypotension as a function of the chosen definition: Literature definitions applied to a retrospective cohort using automated data collection. Anesthesiology 107: 213-220.

13. Choi MH, Chae JS, Lee HJ, et al. (2020) Pre-anaesthesia ultrasonography of the subclavian/infraclavicular axillary vein for predicting hypotension after inducing general anaesthesia: A prospective observational study. Eur J Anaesthesiol 37: 474-481.

14. Sudfeld S, Brechnitz S, Wagner JY, et al. (2017) Post-induction hypotension and early intraoperative hypotension associated with general anaesthesia. Brit J Anaesth 119: 57-64.

15. van KleiWA, van Waes JA, Pasma W, et al. (2017) Relationship between preoperative evaluation blood pressure and preinduction blood pressure: A cohort study in patients undergoing general anesthesia. Anesth Analg 124: 431-437. 
Citation: Bernardis RCGD, Souza HKVD, Izquierdo JAl, et al. (2020) Relationship among Preoperative, Pre and Postinduction Blood Pressure in Normotensive and Hypertensive Patients. J Clin Anesth Pain Manag 4(2):143-149

16. Drummond JC, Blake JL, Patel PM, et al. (2013) An observational study of the influence of "white-coat hypertension" on day-ofsurgery blood pressure determinations. J Neurosurg Anesthesiol 25: 154-161.

17. Saugel B, Reese PC, Sessler DI, et al. (2019) Automated ambulatory blood pressure measurements and intraoperative hypotension in patients having noncardiac surgery with general anesthesia. Anesthesiology 131: 74-83.

18. Vernooij LM, van Klei WA, Machina M, et al. (2018) Different methods of modelling intraoperative hypotension and their association with postoperative complications in patients undergoing non-cardiac surgery. Br J Anaesth 120: 1080-1089.

19. Levin MA, Fischer GW, Lin HM, et al. (2015) Intraoperative ar- terial blood pressure lability is associated with improved 30 day survival. Br J Anaesth 115: 716-726.

20. Schonberger RB, Burg MM, Holt N, et al. (2012) The relationship between preoperative and primary care blood pressure among veterans presenting from home for surgery: Is there evidence for anesthesiologist-initiated blood pressure referral? Anesth Analg 114: $205-214$

21. Khan Al, Fischer M, Pedoto AC, et al. (2020) The impact of fluid optimisation before induction of anaesthesia on hypotension after induction. Anaesthesia 75: 634-641.

22. Dai S, Li X, Yang Y, et al. (2020) A retrospective cohort analysis for the risk factors of intraoperative hypotension. Int J Clin Pract 74: e13521. 